\title{
Performance Evaluation of Gait Recognition using the Largest Inertial Sensor-based Gait Database
}

\author{
Ngo Thanh Trung ${ }^{a}$, Yasushi Makihara ${ }^{a}$, Hajime Nagahara $^{b}$, Yasuhiro Mukaigawa $^{a}$, and Yasushi Yagi ${ }^{a *}$
}

\begin{abstract}
This paper presents the largest inertial sensor-based gait database in the world and its application to a statistically reliable performance evaluation for gait-based recognition problem. Whereas existing gait databases include at most a hundred subjects, we construct a much larger gait database for both accelerometer and gyroscope which includes 736 subjects (382 males and 354 females) with ages ranging from 2 to 78 years. Because a sufficiently large number of subjects for each gender and age group are included in this database, we can analyze the dependence of gait recognition performance on gender or age groups. The results with the latest existing recognition method provide several novel insights, such as the trade-off of gait recognition performance among age groups derived from the maturity of walking ability and physical strength. Moreover, the evaluation for the recognition performance improvement with a larger number of subjects was reliably confirmed in the experiments. As for sensor data type, acceleration is better than angular velocity for gait recognition performance. Compared to unnormalized distance (such as Euclidean distance), normalized distance (such as normalized cross correlation-based distance) works significantly better for angular velocity.
\end{abstract}

\section{Introduction}

Recently, smart wearable or portable electronics are being developed so rapidly, and they are expected to be sophisticated enough in the future to be able to interact with the owner and understand his/her needs, intentions, and health conditions [2,3]. Inertial sensor (gyroscope or accelerometer) is in fact increasingly being embedded in commercial portable electronic devices such as smart phones due to their high cost performance, and inertial sensor-based owner assistance, such as user recognition, from mobile phones is an active research topic.

There are many existing methods to recognize the person

$* a$ The Institute of Scientific and Industrial Research, Osaka University, Japan,

${ }^{b}$ Kyushu University, Japan who carries the sensor based on gait using an inertial sensor such as identification $[1,21,17,18,15]$ or authentication $[9,10,13,24,25,5,18,28]$, which showed the promising applications of inertial sensor for biometric recognition. In these research works, the authors evaluated their methods with a small number of subjects. Their databases are limited in age variety and biased in gender. Therefore, the reliability of the performance evaluation is limited.

Therefore, in this paper, we present a large database for the reliable performance evaluation. In fact, it is the largest inertial sensor-based gait database in the world. The advantages of the database includes: (1) very large number of subjects (736), (2) the gender ratio is close to 1, (3) wide age range (2 to 78 years), and (4) both acceleration and angular velocity data. The largest existing gait database comprises at most a hundred subjects with biased distribution of genders and ages. The proposed gait database thus enables us to evaluate gait recognition algorithms in a more statistically reliable way and to reveal how gait recognition performances differ between genders, ages, and inertial sensors.

The outline of this paper is as follows. First, existing gait databases and recognition methods are briefly considered in Section 2. Next, the construction of the largest gait database is addressed in Section 3. Several existing benchmark methods of gait recognition are briefly introduced in Section 4 , with their performance evaluation using the largest gait database presented in Section 5. Section 6 contains our conclusions and future work in the area.

\section{Related Work}

In this section, we first describe about the existing databases in this research field and then describe the existing methods on the inertial sensor-based gait recognition.

\subsection{Existing inertial gait databases}

There are many databases have been used in this field as summarized in Table 1, however they are not published as the standard databases for the research community.

The largest existing database was used by Gafurov et al. [12] with 100 subjects, the gender is biased with number of males is twice larger than that of females. This 
Table 1. Existing inertial gait databases

\begin{tabular}{|c|c|c|c|}
\hline Method & \#Subjects & Age/Gender variation & Sensor description \\
\hline$\overline{\text { Gafurov et al. }[12,4,8] .}$ & 100 & 70 males, 30 females, ages of 19 to 62 & Accelerometer, hip, 4 sequences/subject \\
\hline Derawi et al. [5] & 60 & NA (Not Available) & Accelerometer, hip, 12 sequences/subject \\
\hline Kobayashi et al. [17] & 58 & NA & $\begin{array}{l}\text { Accelerometer, hand-held, } 40 \text { se- } \\
\text { quences/subject }\end{array}$ \\
\hline Derawi et al. [6] & 51 & $\begin{array}{l}41 \text { males and } 10 \text { females at ages of } 20 \mathrm{~s} \text { and } \\
30 \mathrm{~s}\end{array}$ & 2 days $\times 2$ sequences/ subject \\
\hline Gafurov [11] & 50 & NA & Accelerometer, trousers pocket \\
\hline Jennifer et al. [18] & 36 & NA & Accelerometer, pocket \\
\hline Ailisto et al. $[1,21]$ & 36 & Adults, 17 females, 19 males & $\begin{array}{l}\text { Waist, accelerometer, different days, fast, } \\
\text { slow, normal speeds }\end{array}$ \\
\hline Rong et al. [24] & 35 & $\begin{array}{l}19 \text { male, } 16 \text { female, aging between } 20 \text { and } 45 \\
\text { years old }\end{array}$ & Accelerometer, 5 sequences/subject \\
\hline Trung et al. [28] & 32 & $\begin{array}{l}7 \text { females and } 25 \text { males, ages of } 21 \text { to } 40 \\
\text { years old }\end{array}$ & $\begin{array}{l}\text { Gyroscope, } 5 \text { sequences/subject with differ- } \\
\text { ent attached weights }\end{array}$ \\
\hline Vildjiounaite et al. [29] & 31 & 19 males and 12 females & $\begin{array}{l}2 \text { sequences/subject, accelerometer, hip } \\
\text { pocket, breast pocket and hand-held }\end{array}$ \\
\hline Rong et al. [25] & 21 & $\begin{array}{l}10 \text { males and } 11 \text { females at ages between } 19 \\
\text { and } 40\end{array}$ & $\begin{array}{l}\text { Accelerometer, waist at center back } 5 \text { se- } \\
\text { quences on different days }\end{array}$ \\
\hline Huang et al. [14] & 9 & NA & $\begin{array}{l}\text { Both accelerometer and gyroscope attached } \\
\text { on shoe }\end{array}$ \\
\hline Our database & 736 & $\begin{array}{l}382 \text { males and } 354 \text { females, ages ranging } \\
\text { from } 2 \text { to } 78 \text { years }\end{array}$ & $\begin{array}{l}\text { Waist, both accelerometer and gyroscope, } 2 \\
\text { sequences/subject }\end{array}$ \\
\hline
\end{tabular}

database was frequently used to evaluate the algorithms in the same research group $[12,4,8,7]$. This database supplies quite a large variation of age and gender for reliable performance evaluation and they reached an performance evaluation of 13\% EER (Equal Error Rate) [12]. Derawi et al. [5], Kobayashi et al. [17], Gafurov et al. [11,7] and Jennifer et al. [18] evaluated their method with databases of 60, 58, 50 and 36 subjects, respectively, without age and gender information. Derawi et al. [6] again captured another dataset of 51 subjects with very biased gender and age.

In overall, the number of subjects in these databases is insufficient for statistically reliable performance evaluation. In addition, age variety is limited to adult and/or gender is biased.

\subsection{Recognition methods}

Because the human gait is a periodic motion, most methods of gait-based recognition extract frequency-domain features or detect periods (walking cycles) for constructing gait patterns.

Some researchers used frequency-domain features such as a histogram of intensity $[9,18]$ or the coefficients of Fourier transform [21, 17]. To obtain such frequencydomain features, we need a relatively long and stable gait signal. However, a real walking signal is very noisy, temporally distorted and sensor pose may change, and hence such a long stable gait signal is rarely available. Therefore, frequency-based techniques are outperformed by walking period detection-based methods in most of the sit- uations [25, 28]. Moreover, period detection-based methods can be applied a single period signal (about 1 second), which implies potential real-time applications, while the frequency-based methods cannot be applied.

A large number of recognition systems $[21,9,1,10,27$, $13,15,24,25,5,28]$ detect the walking period, which contains the motion signals of both the steps of the left and right legs, to construct a gait pattern for recognition. Gait period can be detected using heuristic information on the gait signal [1, 24, 5, 13] or without heuristic information [28].

In the experiment, we select some latest methods based on gait period detection for evaluation with the proposed database.

\section{Gait Database Construction}

\subsection{Setup of gait capture system}

The sensor and view of the capture system are shown in Figure 1(a), and (b), respectively. A subject had to walk within the straight white lines $9 \mathrm{~m}$ long. The inertial sensor, named IMU-Z [22], which is shown in Figure 1(a), was fixed at the center of the back waist of the subject by a belt, as shown in Figure 1(b). It was connected to a remote computer by Blue-tooth connection and captured data at framerate of $100 \mathrm{~Hz}$. An IMU-Z includes a triaxial accelerometer, a triaxial gyroscope, and a triaxial compass. The sensor and belt were covered by a soft cushion to protect the sensor and avoid the direct contact to the subject. 


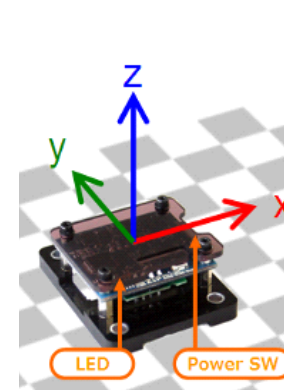

(a) IMU-Z

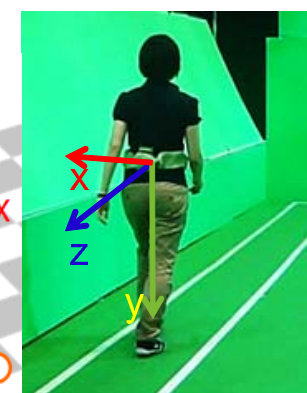

(b) IMU-Z is attached at the center of the back waist

Figure 1. Setup of the gait capturing system.

\subsection{Database information}

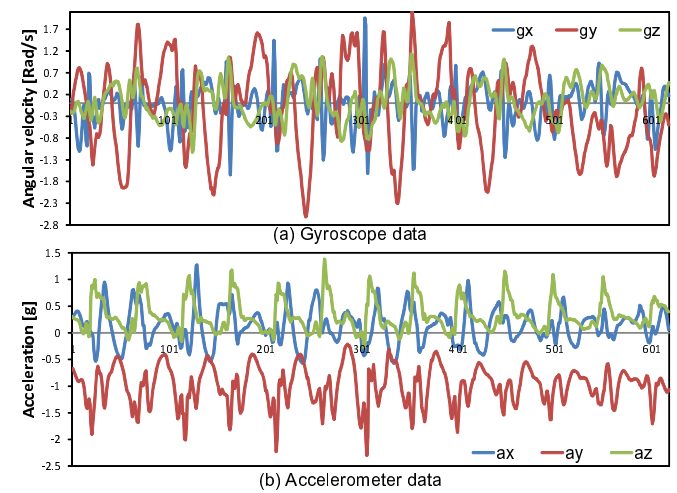

Figure 2. Example of gait signals from (a) gyroscope and (b) accelerometer for a subject in the straight path.

We collected the gait data of visitors in an exhibition during five days. Each subject was requested to sign an informed consent to permit the use of their data for research purpose. He/She also supplied the basic personal information such as gender and age. Thanks to them, we got the world largest database on inertial sensor-based gait.

We captured 6D signal sequences from accelerometer and gyroscope in the IMU-Z sensor. Each subject was asked to walk twice, then two sequences were captured. An example of a 6D signal sequence of a subject is separately shown in Figure 2 for (a) accelerometer and (b) gyroscope, it is about 6 seconds long.

After simple preprocessing to remove invalid data, we had a collection of 736 subjects (382 males and 354 females) with ages ranging from 2 to 78 years. The details description can be found in Figure 3. Compared with the existing databases, the advantages of this database are as follows.

1. The number of subjects is approximately 7 times more than in the existing largest gait databases. This significantly improves the reliability of the gait recognition performance evaluation.

2. The male-to-female ratio is close to 1 . This is a desirable property for more reliable performance evaluation

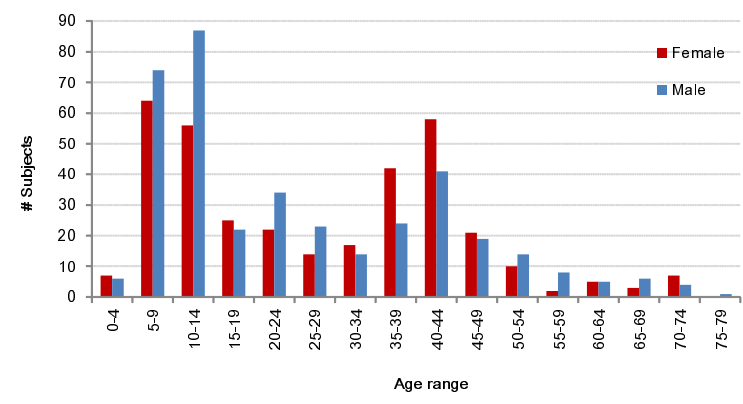

Figure 3. Distribution of age and gender of the database.

of gait-based gender classification and for comparison of gait recognition performance between genders.

3. The subjects' ages are widely distributed from 2 to 78 years. In particular, the number of children is comparable to the number of adults. This provides more statistically reliable results on gait-based age group classification and difficulty level comparison of gait recognition among age groups.

4. 6D gait signal includes 3D acceleration and 3D angular velocity data captured at high frame rate is not only useful for gait recognition but also for understanding the walk motion.

\section{Benchmark Methods}

To evaluate this large database, we apply recent four benchmark methods by Rong et al. [24], Gafurov et al. [13], Derawi et al. [5], and Trung et al. [28]. We briefly summarize these methods in this section, the details can be found in the reference papers. The comparison between these methods can be also found in [28] for gyroscope, the number of subjects is, however, limited to just 32 .

\subsection{Gait period detection}

Gait period detection is a key procedure for gait recognition and the methods differ among the benchmark methods.

Rong et al. [24] detect periods by using up-down acceleration after normalizing the data to range $[-1,+1]$. Wavelet denoising is first applied, and local minimum points are then detected. Zero-cross point is detected just after a local minimum point. Next, four consecutive zero-cross points make a gait period. Finally, a fixed-size pattern is constructed by omitting samples so as to remain the maximum signal intensity difference within a period.

Gafurov et al. [13] detect periods by using the up-down acceleration after being adjusted to be zero-mean. A start point of a gait period is found by the zero-cross point (from negative to positive), then autocorrelation was used to estimate the period length. The start point of the next period is the end point of the previous. 
Derawi et al. [5] detect periods by using the resultant signal (magnitude of 3D acceleration signal). First, the authors extract a single sub-sequence of the signal with a fixed length, and then slide the sub-sequence along the signal to compute the matching distances and find the local minimum points of this distance sequence. These local minimum points are combined with the local minimum points on resultant signal to refine the period locations. All the gait periods can then be detected. It is important to have a good sub-sequence, the authors extract the reference subsequence from the middle of the long sequence.

Trung et al. [28] use all the data dimensions for the detection using the phase-registration technique, called Self-DTW [20]. The Self-DTW detects the period using global optimization without using heuristic information on local points. Self-DTW sometimes results in temporally-distorted periods, and then the authors linearize the Time Warping Function (TWF) to correct the distortion. As a result, all detected periods are phase-registered and temporally-undistorted.

In benchmark methods other than Rong et al. [24], fixedsize pattern is constructed by simple re-sampling based on the detected period.

\subsection{Recognition}

First, a gallery $\mathbb{G}$ is defined as a collection of all the sample patterns for the owner: $\mathbb{G}=\left\{\boldsymbol{g}_{i}\right\}, \boldsymbol{g}_{i}=\left\{g_{i, j} \mid j=\right.$ $0 . . N-1\}$, where $N$ is size of a pattern. A circularly shifted gallery pattern $\boldsymbol{g}_{i}$ by $s$ is defined $\boldsymbol{g}_{i}^{s}=\left\{g_{i,(j+s) \bmod N}\right\}$.

For any probe pattern $\boldsymbol{p}=\left\{p_{j}\right\}$, the distance between it and all the shifted patterns in $\mathbb{G}$ is computed using the min rule [16]:

$$
\operatorname{Dist}(\mathbb{G}, \boldsymbol{p})=\min _{i, s} D\left(\boldsymbol{g}_{i}^{s}, \boldsymbol{p}\right),
$$

where $D(.,$.$) is the distance between \boldsymbol{g}_{i}^{s}$ and $\boldsymbol{p}$.

Gafurov et al. [13] use the Euclidean distance (L2 norm) for $D(.,$.$) . In the other benchmark methods [24,5,28]$, the distance $D(.,$.$) is computed as the normalized cumulative$ DTW score based on Euclidean distance at the end of the optimal warping path.

In fact, some other distance functions such as normalized cross correlation (NCC), Manhattan distance (L1 norm), Tanimoto distance [19] can also be used. Therefore, we will evaluate these four distance measures, two normalized distance measures (NCC and Tanimoto) and two unnormalized distance measures (L1 and L2 norm) in Section 5.4.

\section{Performance Evaluation}

\subsection{Evaluation procedure}

In the experiments, we used all our extracted gait data. Each signal sequence was divided equally into probe and gallery data for the personal authentication scenario. Data from gyroscope and accelerometer was evaluated separately, but period detection was done together.

Recognition performance was evaluated by Receiver Operating Characteristic (ROC) curve [23]. The ROC curve denotes a trade-off curve between a False Rejection Rate (FRR) and False Acceptance Rate (FAR) when the acceptance threshold is changed by a receiver in personal authentication scenario. The Equal Error Rate (EER) where FRR and FAR are equal was also used to evaluate the performance. The lower the EER, the better the method performs.

\subsection{Impact of the number of subjects}

In this section, the impact of the number of subjects is investigated. Based on a statistical analysis of ROC curves [26], the standard deviation of the observed FRR $\hat{p}$ with multiple attempts for each subject is estimated as

$$
\hat{\sigma}(\hat{p})=\sqrt{\frac{\sum a_{i}^{2}-2 \hat{p} \sum a_{i} m_{i}+\hat{p}^{2} \sum m_{i}^{2}}{m^{2} n(n-1)}},
$$

where these variables are described as follows:

- $a_{i}$ : the number of false rejections for $i^{t h}$ subject

- $m_{i}$ : the number of probe samples from $i^{\text {th }}$ subject

- $m$ : the average number of probe samples per subject

- $n$ : the number of subjects

This indicates that the obtained FRR becomes more reliable as the number of subjects increases. To validate the estimation, we repeated the experiments for acceleration data with randomly chosen subsets of fewer subjects and compared the actual standard deviation of the performance and the estimated one from Equ. (2). First, we prepared 20 subsets comprising 100 subjects randomly chosen from the whole set and obtained 20 ROC curves for the benchmark method [28] with NCC distance measure from the experimental results. Then, we calculated the average and standard deviation of the FRR for each FAR, depicted as an averaged ROC curve (blue line) and standard deviation range bar (gray bar) in Figure 4. In addition, the estimated standard deviation range from Equ. (2) is depicted as blue dashed lines. From this graph, we can see that the standard deviation ranges derived from the experimental results correspond well with those estimated from Equ. (2).

Moreover, the results for the whole set are superimposed as a red line, while the standard deviation range estimated from Equ. (2) is depicted as two red dashed lines in Fig. 4. We can see that the standard deviation range is significantly narrower than that of the smaller subject subsets. For example at $4 \%$ FAR, the standard deviation is reduced from $1.9 \%$ to $0.7 \%$. This indicates that the accuracy of the performance evaluation increases approximately three fold. 


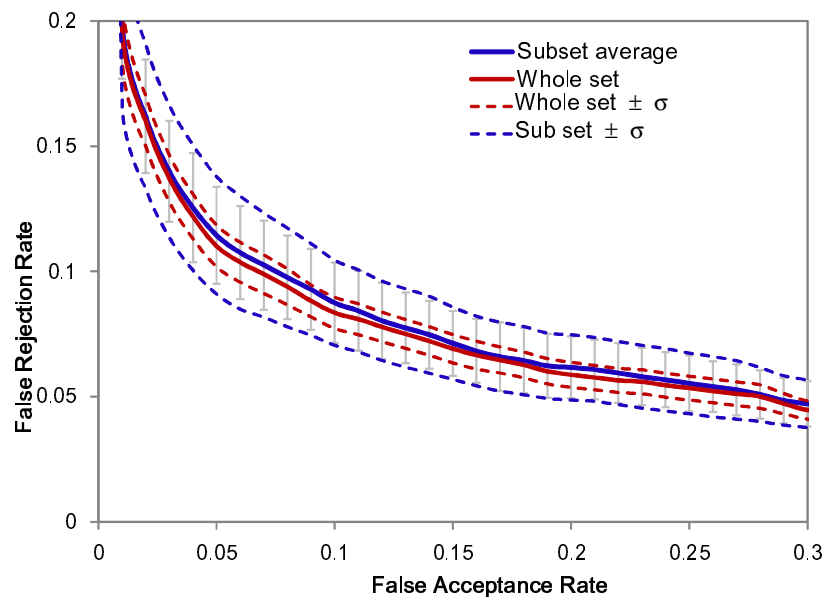

Figure 4. ROC curves with different numbers of subjects. Red and blue stand for the whole set and smaller subsets. A line and two bounding dashed lines of the same color mean a false rejection rate $p$ and its theoretical standard deviation range $p \pm \sigma$ derived from Equ. (2). Gray bars are standard deviation ranges $p \pm \sigma$ obtained by experiments.

\subsection{Evaluation on benchmark methods}

In this experiment, we evaluated the performance of the benchmark methods described in Section 4. All methods computed the pattern distance based on Euclidean distance measure, as described in Section 4.2.

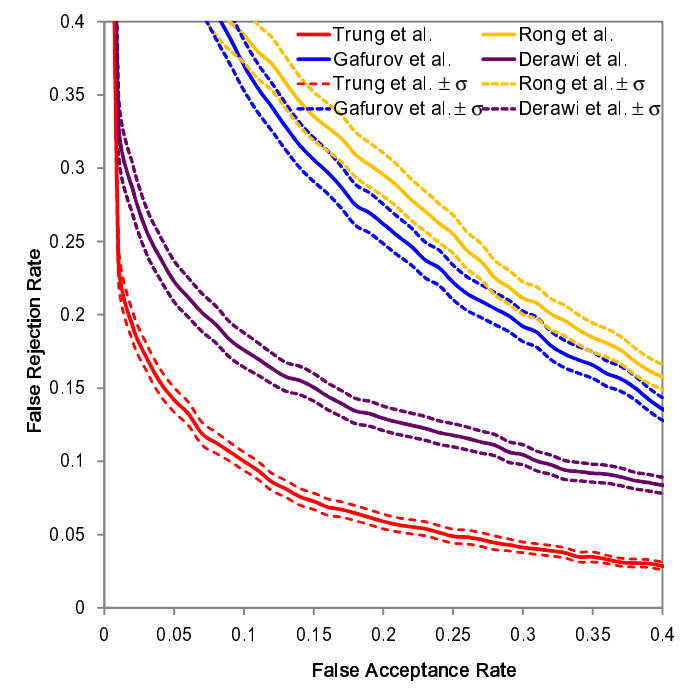

Figure 5. Evaluation for benchmark methods for accelerometer. A line and two dashed boundaries of the same color mean a false rejection rate $p$ and its theoretical standard deviation range $p \pm \sigma$ derived from Equ. (2).

The ROC curves and their theoretical standard deviation of the benchmark methods are shown in Figure 5 for accelerometer and full database. We can see that the evaluation for these benchmark methods is reliable with our database since the deviation boundaries for these ROC curves are clearly separated. However, if the small number of subjects was used, we cannot positively confirm the

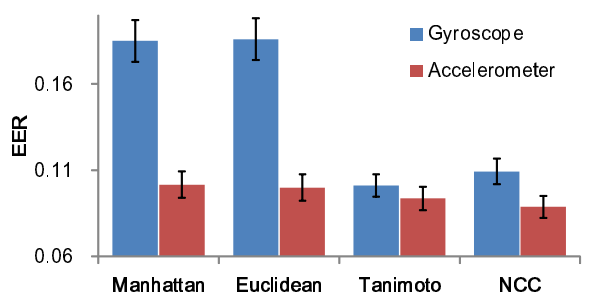

Figure 6. EERs for distance functions and sensor types. The black bars denote the standard deviation of FFR at EER estimated by Equ. (2).

reliability since the deviation boundaries are wider. For examples, if we used a small subset of only 100 subjects, these deviation boundaries were about three time wider, as shown in Figure 4.

We also can see that the benchmark method by Trung et al. [28] produced the best result, therefore, we select it for further evaluation in the following sections.

\subsection{Impact of sensor type and distance function}

In this section, using the selected benchmark method [28], we compared the accuracy for using accelerometer and gyroscope, and also explored a proper distance measure for each sensor. Some normalized distances based on NCC and Tanimoto distance were compared with the unnormalized distances, L1 norm (Manhattan distance) and L2 norm (Euclidean distance). The comparison results are shown in Figure 6. From these results, we can see that in overall, accelerometer produces a better recognition performance than the gyroscope. Normalized distance measures (NCC and Tanimoto) works better than unnormalized distance measures (L1 and L2 norms) for the gyroscope's data. Meanwhile, we cannot see a significant difference among all these distance functions for the acceleration data.

These results come from the observation that the interperiod signal fluctuation of the translational motion (acceleration) for a subject when he/she walks straightly forward is smaller than that of the rotational motion (angular velocity). Therefore, the normalization on the angular velocity data is more effective than on the acceleration data.

In the following sections, we select only the acceleration data and NCC distance measure for evaluation.

\subsection{Impact of gender}

In this section, we investigate how gait recognition performance differs between genders. Our large-scale gait database is ideally suited to this since the gender ratio is close to one in total. However, because gender bias still remains for each age group as shown in Fig. 3, we set the same number of males and females from each age group to reduce such bias. There were 6 age groups used in this equalization: under 10, 10s, 20s, 30s, 40s, and over 50 .

The ROC curve and its theoretical deviation for recognition performance among the 349 males are depicted as the 


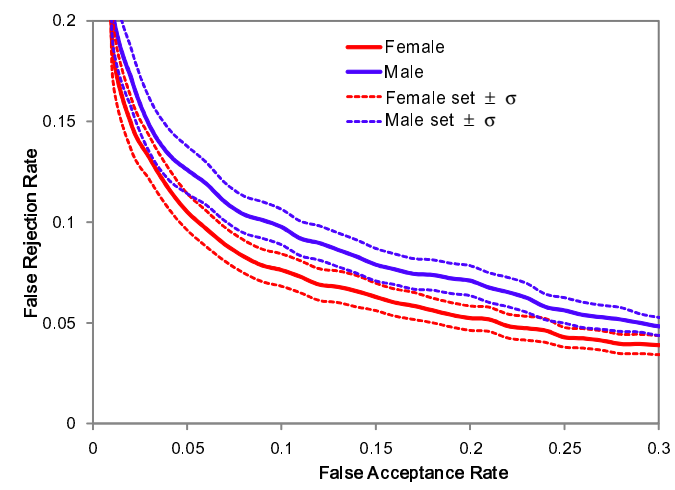

Figure 7. ROC curves of gait recognition for each gender. Blue and red stand for males and females respectively. A line and two dashed boundaries of the same color mean a false rejection rate $p$ and its theoretical standard deviation range $p \pm \sigma$ derived from Equ. (2).

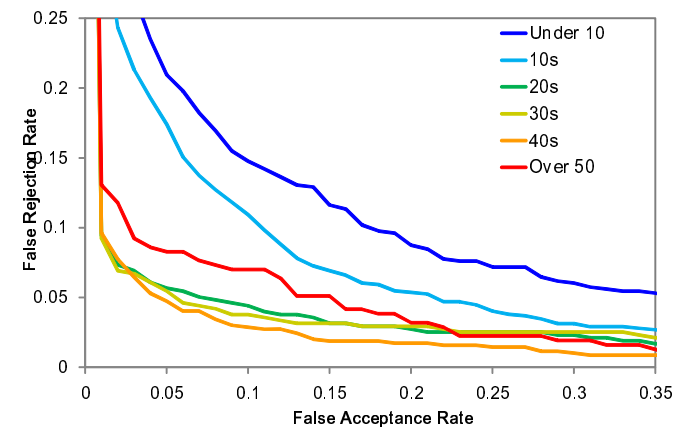

Figure 8. ROC curves of gait recognition for each age-group interval.

blue lines in Figure 7 and those for the 349 females as red lines. According to the results, the recognition performance among females is slightly better than that among males. This evaluation is reliable since the ROCs curves with their deviation boundaries are separable with our database.

\subsection{Impact of age groups}

Next, we show the difference in gait recognition performance among the age groups. Our large-scale gait database is ideally suited to this purpose because the age distribution is much wider than that in existing gait databases.

In the large-scale gait database, each 10-year interval up to 50 years contains about a hundred subjects. The interval over 50 years is treated separately as one age group because of the shortage of subjects. We then separate all the subjects into age groups: under 9, 10s, 20s, 30s, 40s, and over 50. The averaged ROC curves for each age-group interval are depicted in Figure 8.

From this result, we can see that the gait recognition performance for the child age groups is worse than that for the other age groups, and this gradually improves with older groups up to 40 years. This result is understandable because the intra-subject gait fluctuation for children is relatively larger due to the immaturity of their walking skills.
On the other hand, fluctuation in gait for adults is quite small since adults have established their own walking style; in other words, they have a stable gait pattern.

On the other hand, the gait recognition performance for groups over 50 years old declines as the subjects become older. This degradation in performance is inferred from the fact that physical strength generally declines as the subject grows older and hence, the gait tends to fluctuate more.

Consequently, gait recognition performance of subjects in their forties is regarded as a trade-off between the maturity of walking ability and physical strength.

\section{Conclusion}

This paper describes the construction of the world's largest inertial sensor-based gait database and a statistically reliable performance evaluation of inertial sensor-based gait recognition. The database has the following three advantages compared with existing gait databases: (1) the number of subjects is 736, which is approximately 7 times more than existing large-scale gait databases, (2) the male-tofemale ratio is close to one, (3) the age distribution is wide, ranging from 2 to 78 years, and (4) it includes 6D gait signal for both accelerometer and gyroscope. Because sufficient numbers of subjects for each gender and age group are included in the large-scale gait database, it is possible to evaluate how gait recognition performances differ between genders and among age groups.

The results of benchmark recognition method provide several novel insights, such as the trade-off of gait recognition performance among age groups based on the maturity of walking ability and physical strength. Moreover, improvement in the statistical reliability of performance evaluation is shown by comparing the gait recognition results for the whole set and small subsets of a hundred subjects randomly selected from the whole set. The impacts of sensor types and distance functions were also investigated. Accelerometer sensor has better recognition performance than the gyroscope. The normalized distance measure works better than the unnormalized distance measure for gyroscope.

Future work includes performance evaluation of other state-of-the-art gait recognition approaches and present new recognition method for improving the robustness to the sensor attachment. Understanding the age and gender from the gait signals is also one of the attractive research topics.

\section{Acknowledgement}

This work was partially supported by Grant-in-Aid for Scientific Research (S) 21220003, and CREST (Core Research for Evaluation Science and Technology ) of JST (Japan Science and Technology). 


\section{References}

[1] H. J. Ailisto, M. Lindholm, J. Mantyjarvi, E. Vildjiounaite, and S. Makela. Identifying people from gait pattern with accelerometers. In Biometric Technology for Human Identification, SPIE, volume 5779, 2005.

[2] J. C. Augusto and C. D. Nugent. A new architecture for smart homes based on adb and temporal reasoning. In Toward a Human Friendly Assistive Environment, Proceedings of 2 nd International Conference On Smart homes and health Telematic, ICOST2004, pages 106-113, 2004.

[3] D. Cook and S. Das. Smart Environments: Technology, Protocols and Applications. John Wiley and Sons, 2004.

[4] G. D. Security analysis of impostor attempts with respect to gender in gait biometrics. In Biometrics: Theory, Applications, and Systems, First IEEE International Conference on, 2007.

[5] M. O. Derawi, P. Bours, and K. Holien. Improved cycle detection for accelerometer based gait authentication. In Intelligent Information Hiding and Multimedia Signal Processing (IIH-MSP), Sixth International Conference on, 2010.

[6] M. O. Derawi, C. Nickely, P. Bours, and C. Busch. Unobtrusive user-authentication on mobile phones using biometric gait recognition. In Intelligent Information Hiding and Multimedia Signal Processing (IIH-MSP), 2010 Sixth International Conference on, 2010.

[7] D. Gafurov. Performance and Security Analysis. $\mathrm{PhD}$ thesis, Faculty of Mathematics and Natural Sciences at the University of Oslo, 2008.

[8] D. Gafurov and P. Bours. Improved hip based individual recognition using wearable motion recording sensor. In $\mathrm{Se}$ curity Technology, Disaster Recovery and Business Continuity, volume 122 of Communications in Computer and Information Science, pages 179-186. Springer Berlin Heidelberg, 2010.

[9] D. Gafurov, K. Helkala, and T. Sondrol. Biometric gait authentication using accelerometer sensor. Journal of Computers, pages 51-59, 2006.

[10] D. Gafurov and E. Snekkenes. Gait recognition using wearable motion recording sensors. EURASIP Journal on Advances in Signal Processing, 2009.

[11] D. Gafurov, E. Snekkenes, and P. Bours. Gait authentication and identification using wearable accelerometer sensor. In 5th IEEE Workshop on Automatic Identification Advanced Technologies, 2007.

[12] D. Gafurov, E. Snekkenes, and P. Bours. Spoof attacks on gait. IEEE Transactions on Information Forensics and Security, Special Issue on Human Detection and Recognition, 2007.

[13] D. Gafurov, E. Snekkenes, and P. Bours. Improved gait recognition performance using cycle matching. In 2010 IEEE 24th International Conference on Advanced Information Networking and Applications Workshops (WAINA), pages 836-841, 2010.

[14] B. Huang, M. Chen, P. Huang, and Y. Xu. Gait modeling for human identification. In IEEE International Conference on Robotics and Automation, 2007.
[15] A. Kale, N. Cuntoor, B. Yegnanarayana, A. Rajagopalan, and R. Chellappa. Gait analysis for human identification. In Audio- and Video-Based Biometric Person Authentication LNCS, 2003.

[16] J. Kittler, M. Hatef, R. P. Duin, and J. Matas. On combining classifiers. IEEE Transactions on Pattern Analysis and Machine Intelligence, 20(3):226-239, 1998.

[17] T. Kobayashi, K. Hasida, and N. Otsu. Rotation invariant feature extraction from 3-d acceleration signals. In International Conference on Acoustics, Speech, and Signal Processing, pages 3684-3687, 2011.

[18] J. R. Kwapisz, G. M. Weiss, and S. A. Moore. Cell phone based biometric identification. In IEEE Fourth International Conference on Biometrics: Theory, Applications and Systems, 2010.

[19] A. H. Lipkus. A proof of the triangle inequality for the tanimoto distance. Journal of Mathematical Chemistry, 26:263265, 2006.

[20] Y. Makihara, N. T. Trung, H. Nagahara, R. Sagawa, Y. Mukaigawa, and Y. Yagi. Phase registration of a single quasi-periodic signal using self dynamic time warping. In The Tenth Asian Conference on Computer Vision, 2010.

[21] J. Mantyjarvi, M. Lindholm, E. Vildjiounaite, S.-M. Makela, and $\mathrm{H}$. Ailisto. Identifying users of portable devices from gait pattern with accelerometers. In Proc. of IEEE International Conference on Acoustics, Speech, and Signal Processing, 2005.

[22] ZMP Inc. IMU-Z. http://www.zmp.co.jp/e-nuvo/jp/imuz.html, In Japanese.

[23] P. Phillips, D. Blackburn, M. Bone, P. Grother, R. Micheals, and E. Tabassi. Face recogntion vendor test. http://www.frvt.org, 2002.

[24] L. Rong, Z. Jianzhong, L. Ming, and H. Xiangfeng. A wearable acceleration sensor system for gait recognition. In 2nd IEEE Conference on Industrial Electronics and Applications, pages 2654-2659, 2007.

[25] L. Rong, D. Zhiguo, Z. Jianzhong, and L. Ming. Identification of individual walking patterns using gait acceleration. In The 1st International Conference on Bioinformatics and Biomedical Engineering, pages 543-546, 2007.

[26] G. SNEDECOR and W. COCHRAN. Statitical methods. Iowa State University Press, 1967.

[27] S. Sprager and D. Zazula. A cumulant-based method for gait identification using accelerometer data with principal component analysis and support vector machine. WSEAS Transactions on Signal Processing, 5(11), 2009.

[28] N. Trung, Y. Makihara, H. Nagahara, R. Sagawa, Y. Mukaigawa, and Y. Yagi. Phase registration in a gallery improving gait authentication. In the International Joint Conference on Biometrics (IJCB2011). IEEE and IAPR, 2011.

[29] E. Vildjiounaite, S.-M. Makela, M. Lindholm, R. Riihimaki, V. Kyllonen, J. Mantyjarvi, and H. Ailisto. Unobtrusive multimodal biometrics for ensuring privacy and information security with personal devices. In Pervasive Computing, 4th International Conference, PERVASIVE, pages 187-201, 2006. 